\title{
SÍNDROME DE PRUNE BELLY
}

\section{PRUNE BELLY SYNDROME}

\section{Roni Leonardo Teixeira, ACBC-SP ${ }^{1}$; Alexandre Petreca ${ }^{1}$; Patrícia Andréa Torres Garcia ${ }^{1}$}

\section{INTRODUÇÃO}

A Síndrome de Prune-Belly ("abdome em ameixa seca"), é uma forma de uropatia fetal , sem etiologia definida, com uma incidência que varia de 1/35000 à 1/50000 nascidos vivos, sendo caracterizada por uma tríade clássica : ausência, deficiência ou hipoplasia congênita da musculatura da parede abdominal, criptorquidia bilateral e anormalidades do trato urinário.

\section{RELATO DO CASO}

$\mathrm{RN}$, masculino, com 21 dias de vida é internado com história de "micção pelo umbigo" desde o nascimento, sem outros sinais e/ou sintomas associados. Segundo a família, a criança nasceu de parto cesáreo a termo, com seguimento pré-natal regular e sem intercorrências. Ao exame físico, notou-se abdome em "batráquio", com ausência da musculatura abdominal, presença de orifício em cicatriz umbilical drenando urina (Figura 1) e ausência de testículos palpáveis. Demais segmentos não apresentavam alterações. Exames laboratoriais mostraram função renal normal, infecção urinária confirmada por exames de urina (urocultura: pseudomonas sp.) e leucocitose. Havia febre e saída de secreção purulenta pelo úraco.

Ultra-sonografia de vias urinárias mostrou acentuada ureterohidronefrose bilateral com tortuosidade ureteral, além de bexiga alongada e vazia. Fistulografia confirmou a persistência do úraco, refluxo vésico-ureteral grau $\mathrm{V}$ bilateral,

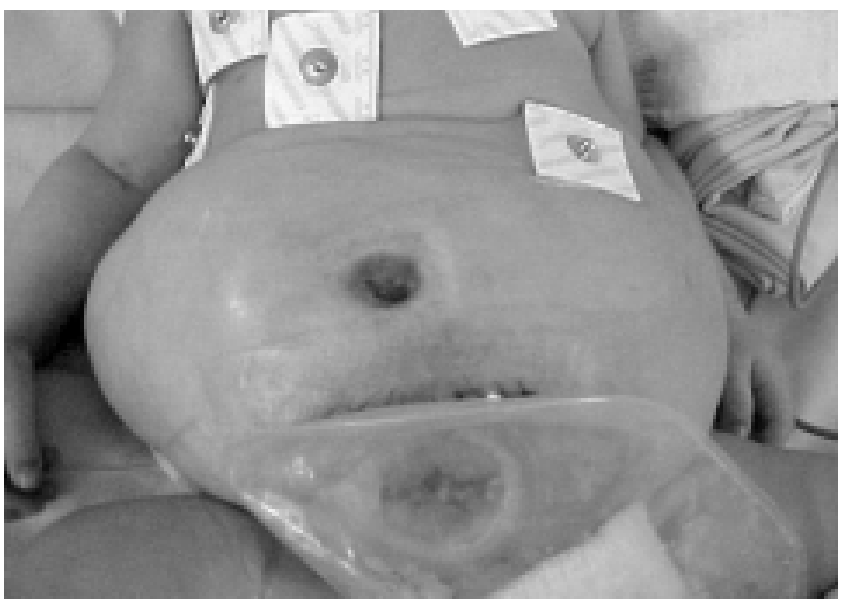

Figura 1 - Criança com "abdome em ameixa seca”, apresentando micção por cicatriz umbilical. com dilatação e tortuosidade ureteral, mostrando uretra de morfologia normal. A cintilografia renal estática (DMSA) mostrou fluxo e função renal normais bilateralmente, hidronefrose, acentuada dilatação e tortuosidade ureteral bilateralmente, com resposta insatisfatória após diurético (achado com baixa especificidade devido acentuada dilatação ureteral).

A opção cirúrgica adotada foi a ressecção do úraco, com confecção de vesicostomia em um primeiro tempo, com intuito de evitar infecções urinárias recorrentes, já que o tratamento definitivo ficou para quando a criança atingir os doze meses de vida , quando terá maior peso para submeter-se a uma agressão cirúrgica maior, consistindo de reimplante ureteral bilateral, orquidopexia bilateral, fechamento de vesicostomia e, após alguns anos, a abdominoplastia. Mantém-se em seguimento ambulatorial, assintomático, sem alterações clínicas e /ou laboratoriais, em uso de antibioticoprofilaxia (nitrofurantoína $5 \mathrm{mg} / \mathrm{Kg} / \mathrm{dia}$ ), com retornos bimestrais programados até o primeiro ano de vida.

\section{DISCUSSÃO}

A síndrome de Prune-Belly é causada por um possível defeito à nível da placa intermédio-lateral do mesoderma ou da proeminência genital . Quanto à associação com a persistência de úraco, é um fato raro, sendo a mais incomum das mal-formações do trato urinário nesta síndrome ${ }^{1}$. O diagnóstico pode ser firmado ainda intra-útero, por volta da $13^{\circ}$ semana, com a evidência de megacistos , megabexiga, ascite fetal, poli ou oligohidrâmnio, fato não observado neste caso. Outras malformações podem estar presentes junto à tríade clássica, dentre elas, as cardíacas, correspondendo a $10 \%$ dos casos, e ainda, as gastrointestinais, tais como má rotação intestinal, entre outras. Nosso paciente possuía somente as alterações inerentes à tríade.

A evolução clínica está diretamente relacionada às complicações e às malformações associadas e na maioria dos casos, os pacientes desenvolvem complicações urinárias e/ ou respiratórias. A recorrência de infecções urinárias, pode levar à insuficiência renal e óbito. Normalmente, os indivíduos que sobrevivem são estéreis, apesar da função hormonal testicular estar normal ${ }^{5}$. Os resultados das cirurgias reconstrutivas é limitado pela qualidade das estruturas do trato urinário. A realização de nefrostomia ou cistostomia, aumenta a possibilidade de infecções urinárias, devido à presença de sondas. Uma vesicostomia pode ser confeccionada

1. Residente do Departamento de Cirurgia Pediátrica da Faculdade de Medicina de São José do Rio Preto - SP.

Recebido em 28/01/2004

Aceito para publicação em 17/02/2004

Trabalho realizado no Hospital de Base da Faculdade de Medicina de São José do Rio Preto - SP. 
com sucesso em pacientes com Prune-Belly ( Figura 2), mantendo uma drenagem satisfatória ${ }^{2}$, conduta esta adotada por nosso Serviço , evitando infecções recorrentes e sepse.

A resolução rápida da uropatia fetal é mandatória, e em alguns casos é realizada intra-útero, através de shunt vesicoamniótico, no segundo trimestre de gestação, após cariotipagem normal, exclusão de malformações à ultrasonografia e por fim, função renal normal com análises seriadas da urina fetal ${ }^{4}$. A orquidopexia é confeccionada segundo a técnica clássica de Fowler-Stephens, concomitante ao reimplante ureteral e fechamento da vesicostomia ${ }^{3}$. Devido ao melhor campo cirúrgico e maior trauma, opta-se por realizar a cirurgia por volta dos seis meses a um ano de idade. Com relação à abdominoplastia, a plicatura extra-peritoneal é uma das técnicas mais usadas, porém, o procedimento deve ser evitado nas fases de crescimento, devido ao desenvolvimento de distúrbios respiratórios por tração abdominal.

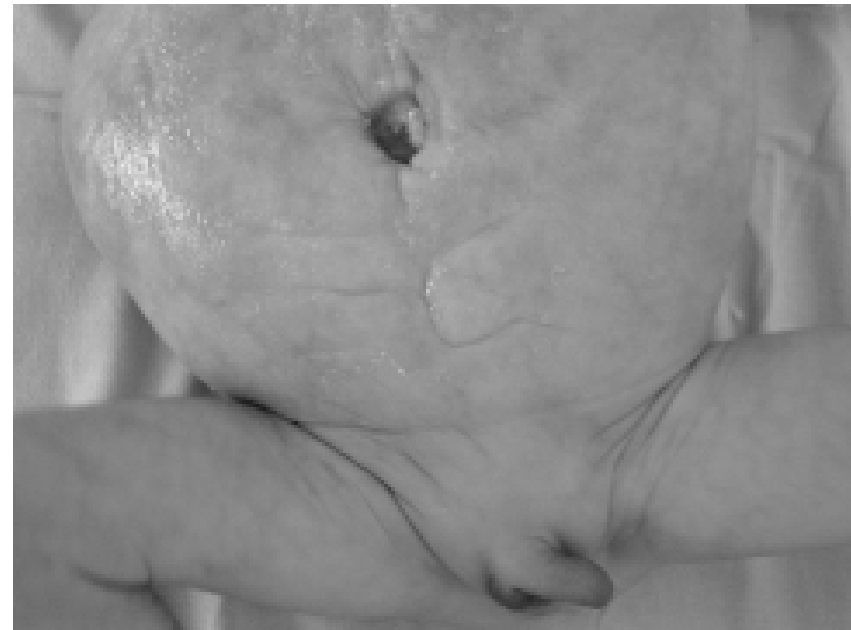

Figura 2 - Vesicostomia confeccionada com úraco patente ressecado.

\begin{abstract}
Prune Belly Syndrome is a fetal uropathy of unknown etiology with incidence of 1/35000 to 1/50000 alive been born, characterized by a classical triad: abdominal musculature congenital deficiency, bilateral criptorquidia and urinary tract malformations. The authors present a case of this rare pathology associated with a patent urachus. After complementary exams confirmed urinary tract alterations (bilateral ureterohidronefrosis and vesicoureteral reflux degree 5, besides urinary infection), the surgical approach was vesicostomy to decrease urinary infections and sepsis. Definitve surgery should be accomplished around the $12^{\text {th }}$ month of life. Nowadays, the child is asymptomatic, with follow-up every two months, with return consultation bimonthly (Rev. Col. Bras. Cir. 2004; 31(6): 400-401).
\end{abstract}

Key words: Prune Belly syndrome; Infant, newborn, diseases; Cystostomy.

\section{REFERÊNCIAS}

1. Cabanillas PL, Albújar PB, Cisneros LI - Síndrome de PrunneBelly. Rev Chil Pediatr, 2001, 72(2):135-138 .

2. Cain MP, Rink RC, Yerkes EB, et al. - Long-term followup and outcome of continent catheterizable vesicostomy using the Rink modification. J Urol, 2002, 168(6):2583-2585.

3. Furness PD, Cheng EY, Franco I, et al. - The prune-belly syndrome: a new and simplified technique of abdominal wall reconstruction. J Urol , 1998, 160(3 Pt 2):1195-1197
4. Leeners B, Sauer I, Schefels J, et al. - Prune-Belly syndrome: therapeutic options including in utero placement of a vesicoamniotic shunt. J Clin Utrasound, 2000, 28(9):500-507

5. Silva AV, Lima FR, Pardi PC - Síndrome de Prune Belly: relato de um caso. Rev Bras Clín Ter, 1999, 25(1):38-41

Endereço para correspondência:

Roni Leonardo Teixeira

Rua Teodoro Demonte, 88/ 202 - Vila São Manoel

15091-260 São José do Rio Preto - SP

E-mail: ronileonardo@zipmail.com.br 